\title{
Identification by mass spectrometry and automated susceptibility testing from positive bottles: a simple, rapid, and standardized approach to reduce the turnaround time in the management of blood cultures
}

\author{
Carola Mauri ${ }^{1}$, Luigi Principe ${ }^{1}$, Silvia Bracco ${ }^{1,2}$, Elisa Meroni ${ }^{1}$, Nicoletta Corbo ${ }^{1}$, Beatrice Pini ${ }^{1}$
}

and Francesco Luzzaro ${ }^{1 *}$ (D)

\begin{abstract}
Background: Speeding up identification and antimicrobial susceptibility testing (AST) is of foremost importance in the management of blood cultures. Here, we describe a simple, rapid, and standardized approach based on a very short-term incubation on solid medium from positive blood cultures followed by MALDI-TOF mass spectrometry identification and automated AST. The aim of the study was to evaluate the impact in the laboratory practice of this new procedure with respect to that previously used (standard method) by comparing TAT and cumulative percentage of final reports to clinicians.

Results: Compared with the standard method, the new procedure gave correct organism identification at genus or species level in $98.4 \%$ of monomicrobial samples. AST resulted in $97.7 \%$ essential agreement and $98.1 \%$ categorical agreement, with $0.9 \%$ minor errors, $1.0 \%$ major error, and $1.5 \%$ very major errors. The mean turnaround time to identification and AST was $61.4 \mathrm{~h}$ by using the new method compared to $83.1 \mathrm{~h}$ by using standard procedure. Concerning cumulative percentages of final reports, approximately a third of results were available at $48 \mathrm{~h}$ from the check-in of the sample when using the new procedure, whereas no final reports were ready at the same time with the standard method.

Conclusions: The new procedure allows faster and reliable results using a simple and standardized approach. Thus, it represents an important tool for a more rapid management of blood cultures when molecular methods are not available in the laboratory.
\end{abstract}

Keywords: Bloodstream infection, Time to positivity, Processing time, Rapid identification, MALDI-TOF, VITEK 2

\footnotetext{
* Correspondence: f.luzzaro@asst-lecco.it

${ }^{1}$ Microbiology and Virology Unit, A. Manzoni Hospital, Lecco, Italy

Full list of author information is available at the end of the article
}

\section{Biomed Central}

(c) The Author(s). 2017 Open Access This article is distributed under the terms of the Creative Commons Attribution 4.0 International License (http://creativecommons.org/licenses/by/4.0/), which permits unrestricted use, distribution, and reproduction in any medium, provided you give appropriate credit to the original author(s) and the source, provide a link to the Creative Commons license, and indicate if changes were made. The Creative Commons Public Domain Dedication waiver (http://creativecommons.org/publicdomain/zero/1.0/) applies to the data made available in this article, unless otherwise stated. 


\section{Background}

Rapid identification and antimicrobial susceptibility results of organisms causing bloodstream infections (BSIs) are an absolute priority for the Microbiology Laboratory. Blood culture is the gold standard method for the diagnosis of BSIs, and it is included among the early activities in the Surviving Sepsis Campaign guidelines [1]. However, it is time-consuming and usually takes on average 24-48 $\mathrm{h}$ for microbial growth in blood culture bottles, and further $48 \mathrm{~h}$ for identification and antimicrobial susceptibility tests after growth in solid culture media [2]. Speeding up these procedures is of foremost importance in the management of bloodstream infections, leading to rapid administration of adequate antimicrobial therapy or adjusting ongoing treatment, and so improving outcome in patients with bacteremia [3]. Reporting Gram stain results of positive blood cultures is useful to guide empiric antimicrobial therapy, but does not provide information about microbial identification at species level [4]. Molecular methods such as real-time polymerase chain reaction (RT-PCR), fluorescent in-situ hybridization (FISH), DNA microarray and nested multiplex PCR have shown to be efficient for the identification of specific microorganisms $[5,6]$. However, molecular methods and related target-based multiplex assays can identify a limited range of microorganisms and antimicrobial resistance genes. Furthermore, molecular methods require additional hands-on processing time and costs [7, 8].

Matrix-Assisted Laser Desorption Ionization Time-ofFlight Mass Spectrometry (MALDI-TOF MS) technology has been introduced as a way to quickly and accurately identify bacteria and yeast $[9,10]$. Compared to standard phenotypic identification, this technology is rapid, inexpensive (after initial purchase of the instrument), and can identify bacteria grown on solid media to the species level [11-13]. Subsequent to the introduction of MALDI-TOF MS for routine diagnostic microbiology, several applications have been developed to further improve and accelerate pathogen identification, especially for bloodstream infections [14, 15]. Particularly, several authors investigated the possibility of direct identification of bacteria from positive blood cultures with MALDI-TOF MS [7, 8, 16-20] while fewer investigated the combined use of MALDI-TOF MS and automated antimicrobial susceptibility testing (AST) instruments [3, 21, 22]. Also, direct identification and AST performed on positive blood culture using massspectrometry and disk diffusion methods, respectively, can provide results about $24 \mathrm{~h}$ earlier than routine standard methods. However, disk diffusion for direct susceptibility testing has not been fully standardized, because it is not related to a specific quantification of the bacterial concentration of the inoculum.
At our Institution, a number of specific approaches have been used to reduce the time of result and improve patient outcome: delivery of blood cultures to the laboratory within one hour, immediate incubation of bottles in the automated instrument and continuous management of blood cultures (365 days/year), communication of preliminary results based on Gram stain (performed at the time of positivity), identification from overnight cultures by MALDI-TOF MS, and direct AST by agar diffusion (using selected antimicrobials as indicators of resistance).

This paper describes a standardized, easy-to-perform and practical approach in order to obtain rapid identification with MALDI-TOF MS and to decrease the time needed to obtain final reports using a standardized bacterial concentration and an automated instrument for definitive susceptibility results. The turnaround time (TAT) was also compared to the standard method to better quantify the impact in the laboratory practice. The aim of the study was to compare these data with those obtained with the previously used workflow of blood cultures.

Part of this work has been presented at the 25th ECCMID meeting, Copenhagen (Denmark), 2015 (ePoster EP-054).

\section{Methods \\ Study design}

The study was organized in two subsequent steps. In the first period, conducted from January to October 2014, positive bottles from blood cultures obtained from different patients were investigated using the current standard routine method in parallel with the new procedure.

In the second period, conducted from November 2014 to September 2015, the new procedure was introduced as the routine method, except for polymicrobial blood cultures. TAT resulting in the second period (i.e., using the new procedure as routine method) was then evaluated in comparison with that obtained in the first period (i.e., using the previous standard method).

Concerning internal workflow, our laboratory runs 24/7 but routine microbiological procedures are performed from 8 am to $5 \mathrm{pm}$. Blood cultures, however, are delivered from wards to the laboratory within one hour from collection and are immediately incubated in the automated instrument at any time (i.e. 24/7). Positive bottles are routinely processed every day within the opening time of the laboratory. Of note, the new procedure was applied 5 days/week (Monday-Friday), from 8 am to $2 \mathrm{pm}$ and only one positive blood culture for each patient was processed. Blood cultures that were found positive from $2 \mathrm{pm}$ to $5 \mathrm{pm}$ were only processed by the standard routine method. Because of limited laboratory staff resources during evening and night hours (from $5 \mathrm{pm}$ to $8 \mathrm{am}$ ), 
samples detected positive during this time were processed the following morning with the new procedure.

\section{Standard identification and antimicrobial susceptibility testing}

Blood samples were inoculated in aerobic and anaerobic non-charcoal based bottles (BacT/ALERT ${ }^{\ominus}$ FA Plus, and FN Plus, respectively), containing adsorbent polymeric beads to neutralize antimicrobials, and incubated in the BacT/ALERT 3D system (bioMérieux, Marcy l'Etoile, France). The standard method for identification and susceptibility testing was performed as described: a small volume of positive blood culture fluid was inoculated on different plates, including Columbia agar with 5\% sheep blood, Chocolate agar with polyvitex, Columbia ANC agar with 5\% sheep blood, Mac Conkey agar, Schaedler agar with Vitamin $\mathrm{K} 1$ and $5 \%$ sheep blood, and Sabouraud agar with gentamicin and chloramphenicol. The inoculated plates were incubated at $36{ }^{\circ} \mathrm{C}$ in $\mathrm{O} 2,5 \%$ $\mathrm{CO} 2$ or anaerobic atmosphere to enable bacterial growth. Gram staining was performed at the time of blood culture positivity detection and immediately communicated by phone to the treating physicians.

Identification was done by MALDI-TOF MS (VITEK ${ }^{\circ}$ MS, bioMérieux). Isolated colonies from agar plates were directly applied to VITEK MS target slide in duplicate (two spots for each isolate) and were covered with one microliter of CHCA ( $\alpha$-Cyano-4-hydroxycinnamic acid) matrix. The identification was considered valid if at least one spot on the target slide gave a confidence level of $\geq 75 \%$ without conflicting identifications from replicate spots of the same sample. A standardized inoculum (McFarland standard of 0.5) was then prepared from single colonies grown on the agar medium, and the appropriate VITEK AST cards (AST-632 for staphylococci, AST-586 for enterococci, AST-01 for streptococcal species other than Streptococcus pneumoniae, AST576 for S. pneumoniae, AST-202 for Gram-negative bacteria), chosen according to the identification results given by the VITEK MS, were inoculated following the manufacturer's recommendations. Antimicrobial susceptibility results were obtained after a 18-24 h incubation using the VITEK $^{\oplus} 2$ (bioMérieux) instrument and interpreted according to current EUCAST criteria [23, 24].

Rapid identification and antimicrobial susceptibility testing subsequent to very short-term incubation on solid medium When a blood culture was flagged positive by the BacT/ ALERT, an aliquot $(2.5 \mathrm{ml})$ of each sample was transferred in a tube with gel separator (BD Vacutainer ${ }^{\odot}$ Blood Collection Tubes) and centrifuged at $3500 \mathrm{rpm}$ for $10 \mathrm{~min}$. The supernatant was discarded and the pellet was inoculated on two blood agar plates and incubated at $36{ }^{\circ} \mathrm{C}$ in $\mathrm{O} 2$ and $5 \% \mathrm{CO} 2$, respectively. After
$3 \mathrm{~h}$ incubation, microbial identification was obtained by VITEK MS directly from bacterial growth on agar plates, as above described. Susceptibility tests were performed even when the rapid identification was available at genus level only. Concurrent with rapid identification, a suspension, adjusted to a McFarland standard of 0.5 , was created from bacterial growth and used for AST on the VITEK 2 instrument as above described.

\section{Data analysis}

Identification and susceptibility results obtained by the new procedure were compared with those obtained with routine standard method. Identification results were classified as correct identification at species or genus level, no identification, and incorrect identification. To compare susceptibility testing results, MICs obtained by both methods were translated in clinical categories as susceptible (S), intermediate (I), or resistant (R) according to the current EUCAST criteria and evaluated in terms of Categorical Agreement (CA), Essential Agreement (EA), Very Major Error (VME, false susceptibility), Major Error (ME, false resistance), and minor Error (mE, susceptible/resistant versus intermediate susceptibility) (International Standard ISO 20776-2) [25]. The Etest method (bioMérieux) was used to resolve interpretation discrepancies.

\section{Application of the new method and evaluation of the turnaround time}

Following the application of the new method, the TAT, calculated as the time from the check-in of the sample to the final report comprehensive of identification and antimicrobial susceptibility test results, was evaluated for the two study periods. It consisted of two components: i) time to positivity, corresponding to the time necessary for the microorganism growth by BacT/ALERT incubation; and ii) processing time, corresponding to the time necessary for generating the final report (including bacterial identification, AST, validation of results, and reporting to clinicians). Cumulative percentages of final reports were also evaluated at different times starting from the check-in of the sample.

Times were calculated using the Microbiology software package (Copernico, bioMérieux) interfaced with the Laboratory Information System (Magellano, Software Team), and evaluated separately for Grampositives and Gram-negatives.

\section{Results}

\section{First study period}

Two hundred and ten blood cultures obtained from different patients were examined during the first period. Compared with standard method, the new procedure allowed to obtain a correct identification at the species level in 187 
(89.0\%) cases (96 Gram-positives and 91 Gram-negatives), whereas $3(1.4 \%)$ isolates were identified at the genus level. Identification at the genus level was obtained for Streptococcus agalactiae $(n=1)$, Salmonella group B $(n=$ $1)$, and Salmonella typhi $(n=1)$. The isolate identified as Salmonella group B was not further investigated at the species level. Overall data are summarized in Table 1. No incorrect identifications were obtained.

Among the 97 Gram-positives, 71 belonged to the genus Staphylococcus, 16 were identified as Enterococcus spp., and 10 were identified as Streptococcus spp.. Of the 93 Gram-negatives, 86 belonged to the family Enterobacteriaceae and 7 were identified as Pseudomonas aeruginosa.

Of the remaining 20 blood cultures, 13 (6.2\%) showed polymicrobial growth, and $3(1.4 \%)$ were regarded as false positives (no growth). VITEK MS was unable to generate an acceptable identification (insufficient peaks) using the new procedure in $4(1.9 \%)$ cases. Isolates not identified were: Staphylococcus hominis $(n=2)$, S. agalactiae $(n=1)$, and Staphylococcus capitis $(n=1)$. Overall, the new procedure gave correct organism identification at genus or species level in $98.4 \%$ of monomicrobial samples.

Only microorganisms identified at species or genus level were studied for AST. A total of 2462 microorganism-antimicrobial combinations were analyzed. Overall, results were as follows: EA, 97.7\% (2405/ 2462); CA, 98.1\% (2414/2462); mE, 0.9\% (21/2462); ME, $1.0 \%$ (20/1940); and VME, 1.5\% (7/478).

Results for Gram-positive bacteria were as follows: EA, 96.9\%; CA, 97.4\%; $\mathrm{mE}$ 0.8\%; ME 1.7\%; and VME, $2.5 \%$ (Table 2). Notably, VME were detected only among coagulase-negative staphylococci (CoNS). Results for

Table 1 Distribution of blood isolates identified in the two study periods

\begin{tabular}{lll}
\hline Microorganism & First study period & Second study period \\
\hline Gram-positive & 30 & 29 \\
S. aureus & 25 & 31 \\
S. epidermidis & 16 & 13 \\
CoNS & 16 & 12 \\
Enterococcus spp. & 10 & 12 \\
Streptococcus spp. & 97 & 97 \\
Total Gram-positive isolates & & \\
Gram-negative & 63 & 68 \\
E. coli & 10 & 8 \\
K. pneumoniae & 7 & 3 \\
P. aeruginosa & - & 2 \\
A. baumannii & 13 & 12 \\
Other Enterobacteriaceae & 93 & 93 \\
Total Gram-negative isolates & 93 \\
Total of isolates & 190 & 190 \\
\hline
\end{tabular}

Gram-negative isolates were: $\mathrm{EA}, 98.4 \%$; $\mathrm{CA}, 98.7 \%$; $\mathrm{mE}$, 0.9\%; and ME, 0.5\% (Table 3). No VME were detected.

Microorganism-antimicrobial combinations that did not result in agreement with conventional methods are listed in Table 4. No discrepancies were observed as regards detection and/or suggestion of the following resistance mechanisms: ESBL production (18/63 Escherichia coli were found to be consistent with ESBL producers); carbapenemase production (2/10 Klebsiella pneumoniae produced KPC-type carbapenemases); methicillin resistance (8/30 Staphylococcus aureus and 25/41 CoNS isolates were methicillin-resistant). With regard to inducible clindamycin resistance (ICR) in staphylococci, 10/30 S. aureus and 7/41 CoNS (S. hominis, $n=5$; Staphylococcus epidermidis, $n=2$ ) were ICR positive with both methods. Three disagreements were observed in CoNS (S. epidermidis, $n=2$, and $S$. hominis, $n=1$ ). In two cases, ICR test was positive with the new procedure but negative with the standard method whereas in the remaining case ICR test was negative with the new procedure but positive with the standard method. The D test consistently confirmed results of the standard method.

\section{Second study period}

One hundred and ninety blood cultures were evaluated during the second period (i.e., after implementing the new method in the laboratory routine). All of them were identified at the species level.

Among the 97 Gram-positives, 73 (75.3\%) belonged to the genus Staphylococcus, 12 (12.4\%) were identified as Enterococcus spp., and 12 (12.4\%) were identified as Streptococcus spp.. Of the 93 Gram-negatives, 88 (94.6\%) belonged to the family Enterobacteriaceae and 5 (5.4\%) were identified as non-fermentative Gramnegatives. Distribution of isolates in this group was similar to that of isolates examined during the first period (Table 1).

\section{Evaluation of the turnaround time}

The mean TAT values were calculated for the two periods in order to compare results obtained using the new procedure with respect to those obtained with the standard method, and are listed in Table 5. The time to positivity was comparable in the two study periods (16.0 h and $16.1 \mathrm{~h}$, using standard and new method, respectively). Using the new approach, the overall mean TAT substantially decreased (from $83.1 \mathrm{~h}$ to $61.4 \mathrm{~h}$ ), especially in the case of Gramnegatives (from $80.4 \mathrm{~h}$ to $56.7 \mathrm{~h}$ ).

Table 6 shows cumulative percentages of final reports available at 48 and $72 \mathrm{~h}$ using the two methods. Of note, no final reports were ready at $48 \mathrm{~h}$ from the check-in of the sample using the standard method, 
Table 2 Comparison of AST results for Gram-positives: agreement of the new rapid procedure with standard method

\begin{tabular}{|c|c|c|c|c|c|c|c|c|c|c|c|c|c|c|}
\hline \multirow[t]{2}{*}{ Antimicrobial agent } & \multirow[t]{2}{*}{ No. of test } & \multicolumn{3}{|c|}{ Susceptibilities } & \multicolumn{2}{|l|}{ EA } & \multicolumn{2}{|l|}{ CA } & \multicolumn{2}{|l|}{$\mathrm{mE}$} & \multicolumn{2}{|l|}{ ME } & \multicolumn{2}{|c|}{ VME } \\
\hline & & $\mathrm{S}$ & I & $R$ & No. & $\%$ & No. & $\%$ & No. & $\%$ & No. & $\%$ & No. & $\%$ \\
\hline Ampicillin & 20 & 18 & 0 & 2 & 20 & 100 & 20 & 100 & 0 & 0.0 & 0 & 0.0 & 0 & 0.0 \\
\hline Ampicillin-sulbactam & 16 & 14 & 0 & 2 & 16 & 100 & 16 & 100 & 0 & 0.0 & 0 & 0.0 & 0 & 0.0 \\
\hline Cefotaxime & 9 & 9 & 0 & 0 & 9 & 100 & 9 & 100 & 0 & 0.0 & 0 & 0.0 & 0 & 0.0 \\
\hline Ceftriaxone & 9 & 9 & 0 & 0 & 8 & 89 & 9 & 100 & 0 & 0.0 & 0 & 0.0 & 0 & 0.0 \\
\hline Clindamycin & 91 & 52 & 0 & 39 & 80 & 88 & 82 & 90 & 2 & 2.2 & 6 & 11.5 & 1 & 2.6 \\
\hline Daptomycin & 71 & 70 & 0 & 1 & 69 & 97 & 70 & 99 & 0 & 0.0 & 1 & 1.4 & 0 & 0.0 \\
\hline Erythromycin & 95 & 48 & 1 & 46 & 93 & 98 & 89 & 94 & 5 & 5.3 & 1 & 1.1 & 0 & 0.0 \\
\hline Gentamicin & 71 & 46 & 0 & 25 & 70 & 99 & 70 & 99 & 0 & 0.0 & 0 & 0.0 & 1 & 4.0 \\
\hline Imipenem & 21 & 20 & 0 & 1 & 21 & 100 & 21 & 100 & 0 & 0.0 & 0 & 0.0 & 0 & 0.0 \\
\hline Levofloxacin & 95 & 54 & 1 & 40 & 95 & 100 & 94 & 99 & 1 & 1.1 & 0 & 0.0 & 0 & 0.0 \\
\hline Linezolid & 92 & 92 & 0 & 0 & 91 & 99 & 92 & 100 & 0 & 0.0 & 0 & 0.0 & 0 & 0.0 \\
\hline Nitrofurantoin & 15 & 15 & 0 & 0 & 15 & 100 & 15 & 100 & 0 & 0.0 & 0 & 0.0 & 0 & 0.0 \\
\hline Oxacillin & 70 & 36 & 0 & 34 & 70 & 100 & 70 & 100 & 0 & 0.0 & 0 & 0.0 & 0 & 0.0 \\
\hline Penicillin & 81 & 23 & 0 & 58 & 78 & 96 & 80 & 99 & 0 & 0.0 & 0 & 0.0 & 1 & 1.7 \\
\hline Rifampicin & 76 & 69 & 0 & 7 & 74 & 97 & 75 & 99 & 0 & 0.0 & 0 & 0.0 & 1 & 14.3 \\
\hline Teicoplanin & 87 & 71 & 0 & 16 & 80 & 92 & 82 & 94 & 0 & 0.0 & 2 & 2.3 & 3 & 18.7 \\
\hline Tetracycline & 76 & 49 & 15 & 12 & 75 & 99 & 74 & 97 & 1 & 1.3 & 1 & 1.3 & 0 & 0.0 \\
\hline Tigecycline & 87 & 87 & 0 & 0 & 87 & 100 & 87 & 100 & 0 & 0.0 & 0 & 0.0 & 0 & 0.0 \\
\hline Vancomycin & 97 & 97 & 0 & 0 & 91 & 94 & 93 & 96 & 0 & 0.0 & 4 & 4.1 & 0 & 0.0 \\
\hline TOTAL & 1179 & 879 & 17 & 283 & 1142 & 96.9 & 1148 & 97.4 & 9 & 0.8 & 15 & 1.7 & 7 & 2.5 \\
\hline
\end{tabular}

whereas $26.5 \%$ of results were available at the same time using the new procedure. At $72 \mathrm{~h}, 37.3 \%$ and $74.6 \%$ of results were available using the standard method and the new procedure, respectively. E. coli and $S$. aureus, i.e., the most frequently isolated organisms for the two groups of Gram-negative and Grampositive bacteria, were separately analyzed. In both cases cumulative percentages of final reports available at 48 and $72 \mathrm{~h}$ using the new procedure were higher than $30 \%$ and $80 \%$, respectively.

Table 3 Comparison of AST results for Gram-negatives: agreement of the new rapid procedure with standard method

\begin{tabular}{|c|c|c|c|c|c|c|c|c|c|c|c|c|c|c|}
\hline \multirow[t]{2}{*}{ Antimicrobial agent } & \multirow[t]{2}{*}{ No. of test } & \multicolumn{3}{|c|}{ Susceptibilities } & \multicolumn{2}{|l|}{ EA } & \multicolumn{2}{|l|}{ CA } & \multicolumn{2}{|l|}{$\mathrm{mE}$} & \multicolumn{2}{|l|}{ ME } & \multicolumn{2}{|l|}{ VME } \\
\hline & & $\mathrm{S}$ & I & $\mathrm{R}$ & No. & $\%$ & No. & $\%$ & No. & $\%$ & No. & $\%$ & No. & $\%$ \\
\hline Amikacin & 93 & 81 & 7 & 5 & 93 & 100 & 92 & 99 & 1 & 1.1 & 0 & 0.0 & 0 & 0.0 \\
\hline Amoxicillin-clavulanate & 92 & 61 & 0 & 31 & 91 & 99 & 89 & 97 & 0 & 0.0 & 3 & 4.9 & 0 & 0.0 \\
\hline Cefepime & 93 & 72 & 7 & 14 & 89 & 96 & 90 & 97 & 3 & 3.3 & 0 & 0.0 & 0 & 0.0 \\
\hline Cefotaxime & 93 & 64 & 0 & 29 & 90 & 97 & 93 & 100 & 0 & 0.0 & 0 & 0.0 & 0 & 0.0 \\
\hline Ceftazidime & 93 & 68 & 6 & 19 & 90 & 97 & 91 & 98 & 2 & 2.2 & 0 & 0.0 & 0 & 0.0 \\
\hline Ciprofloxacin & 93 & 68 & 0 & 25 & 93 & 100 & 93 & 100 & 0 & 0.0 & 0 & 0.0 & 0 & 0.0 \\
\hline Colistin & 89 & 85 & 0 & 4 & 87 & 98 & 89 & 100 & 0 & 0.0 & 0 & 0.0 & 0 & 0.0 \\
\hline Gentamicin & 93 & 84 & 0 & 9 & 93 & 100 & 93 & 100 & 0 & 0.0 & 0 & 0.0 & 0 & 0.0 \\
\hline Ertapenem & 93 & 79 & 1 & 13 & 93 & 100 & 93 & 100 & 0 & 0.0 & 0 & 0.0 & 0 & 0.0 \\
\hline Imipenem & 89 & 86 & 0 & 3 & 89 & 100 & 89 & 100 & 0 & 0.0 & 0 & 0.0 & 0 & 0.0 \\
\hline Meropenem & 93 & 90 & 1 & 2 & 93 & 100 & 93 & 100 & 0 & 0.0 & 0 & 0.0 & 0 & 0.0 \\
\hline Piperacillin-tazobactam & 92 & 79 & 3 & 10 & 88 & 96 & 88 & 96 & 3 & 3.3 & 1 & 1.3 & 0 & 0.0 \\
\hline Tigecycline & 84 & 79 & 2 & 3 & 83 & 99 & 81 & 96 & 3 & 3.6 & 0 & 0.0 & 0 & 0.0 \\
\hline Co-trimoxazole & 93 & 65 & 0 & 28 & 91 & 98 & 92 & 99 & 0 & 0.0 & 1 & 1.5 & 0 & 0.0 \\
\hline TOTAL & 1283 & 1061 & 27 & 195 & 1263 & 98.4 & 1266 & 98.7 & 12 & 0.9 & 5 & 0.5 & 0 & 0 \\
\hline
\end{tabular}


Table 4 Microorganism-antimicrobial combinations that did not result in agreement with standard method ${ }^{\mathrm{a}}$

\begin{tabular}{|c|c|c|c|}
\hline Microorganism & $\mathrm{mE}$ & ME & VME \\
\hline E. cloacae & Tigecycline $(n=1)$ & - & - \\
\hline \multirow[t]{4}{*}{ E. coli } & Piperacillin-tazobactam $(n=3)$ & Amoxicillin-clavulanate $(n=3)$ & - \\
\hline & Cefepime $(n=2)$ & Piperacillin-tazobactam $(n=1)$ & \\
\hline & Ceftazidime $(n=2)$; & Co-trimoxazole $(n=1)$ & \\
\hline & Amikacin $(n=1)$ & & \\
\hline E. faecalis & - & Vancomycin $(n=1)$ & - \\
\hline K. pneumoniae & Tigecycline $(n=2)$ & - & - \\
\hline P. mirabilis & Cefepime $(n=1)$ & - & - \\
\hline \multirow[t]{3}{*}{ S. aureus } & Erythromycin $(n=1)$ & Clindamycin $(n=2)$ & - \\
\hline & Tetracycline $(n=1)$ & Daptomycin $(n=1)$ & \\
\hline & & Vancomycin $(n=1)$ & \\
\hline \multirow[t]{4}{*}{ S. capitis } & Erythromycin $(n=1)$ & Clindamycin $(n=1)$ & - \\
\hline & & Teicoplanin $(n=1)$ & \\
\hline & & Tetracycline $(n=1)$ & \\
\hline & & Vancomycin $(n=1)$ & \\
\hline \multirow[t]{3}{*}{ S. epidermidis } & Clindamycin $(n=2)$ & Clindamycin $(n=2)$ & Teicoplanin $(n=2)$ \\
\hline & Erythromycin $(n=1)$ & Teicoplanin $(n=1)$ & Penicillin $(n=1)$ \\
\hline & & & Rifampicin $(n=1)$ \\
\hline \multirow[t]{3}{*}{ S. hominis } & Levofloxacin $(n=1)$ & Clindamycin $(n=1)$ & Clindamycin $(n=1)$; \\
\hline & & & Gentamicin $(n=1)$ \\
\hline & & & Teicoplanin $(n=1)$ \\
\hline \multirow[t]{2}{*}{ S. pneumoniae } & Erythromycin $(n=2)$ & Erythromycin $(n=1)$; & - \\
\hline & & Vancomycin $(n=1)$ & \\
\hline
\end{tabular}

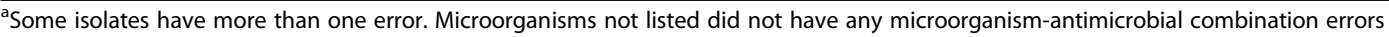

\section{Discussion}

The present study describes a procedure based on a very short-term incubation time on solid medium from positive blood cultures followed by MALDI-TOF MS identification and automated AST (starting from a standardized bacterial concentration) in order to obtain rapid final results.

An early basis for decision making is urgently required in the case of life-threatening conditions such as bloodstream infections [26]. Of note, the International Guidelines for Management of Sepsis and Septic Shock recommend that: i) antimicrobial therapy should be assessed daily for de-escalation; ii) the combination therapy, when used empirically in patients with sepsis, should not be routinely used for ongoing treatment.

Empiric antimicrobial therapy should be narrowed once pathogen identification and sensitivities are established and/or adequate clinical improvement is noted [1]. Molecular methods have shown to be efficient for the rapid identification of specific microorganisms but can identify a limited range of microorganisms and antimicrobial resistance genes, thus partially responding to

Table 5 Mean value of times in the two study periods. First quartile, median and third quartile are reported in brackets

\begin{tabular}{|c|c|c|c|c|c|c|}
\hline \multirow[t]{2}{*}{ Times (in hours) } & \multicolumn{3}{|l|}{ Standard method } & \multicolumn{3}{|l|}{ New procedure } \\
\hline & Total & $\mathrm{GP}^{\mathrm{a}}$ & $\mathrm{GN}^{\mathrm{a}}$ & Total & GP & GN \\
\hline \multirow[t]{2}{*}{ Time to positivity } & 16.0 & 17.9 & 14.1 & 16.1 & 18.9 & 13.3 \\
\hline & $(10.1-14.0-19.1)$ & $(12.1-17.0-22.1)$ & $(10.0-12.0-15.0)$ & $(11.0-13.0-18.1)$ & $(12.1-16.1-22.0)$ & $(10.0-11.1-14.0)$ \\
\hline \multirow[t]{2}{*}{ Processing time } & 67.1 & 67.7 & 66.3 & 45.3 & 47.0 & 43.4 \\
\hline & (52.8-61.2-78.5) & (52.0-59.2-76.2) & $(54.6-62.8-83.4)$ & (31.7-37.7-51.8) & (30.9-37.5-51.9) & $(31.9-37.8-51.3)$ \\
\hline \multirow[t]{2}{*}{ Intra-laboratory TAT } & 83.1 & 85.6 & 80.4 & 61.4 & 65.9 & 56.7 \\
\hline & $(69.3-74.7-93.8)$ & $(69.2-74.8-89.7)$ & $(69.5-74.5-95.6)$ & $(47.6-51.4-72.5)$ & $(48.6-59.8-73.1)$ & $(44.6-50.5-65.6)$ \\
\hline
\end{tabular}

äP: Gram-positives; GN: Gram-negatives 
Table 6 Cumulative percentages of final reports available at 48 and $72 \mathrm{~h}$ from the check-in

\begin{tabular}{llllll}
\hline Microorganism & \multicolumn{2}{l}{$48 \mathrm{~h}$} & & \multicolumn{2}{c}{$\mathrm{h} \mathrm{h}$} \\
\cline { 2 - 3 } \cline { 5 - 6 } & $\mathrm{SM}^{\mathrm{a}}$ & $\mathrm{NP}^{\mathrm{a}}$ & & $\mathrm{SM}$ & $\mathrm{NP}$ \\
\hline Total & 0 & 26.5 & 37.3 & 74.6 \\
Gram-positive & 0 & 19.8 & 36.8 & 71.9 \\
Gram-negative & 0 & 33.3 & 37.8 & 79.6 \\
S. aureus & 0 & 32.1 & 28.6 & 82.1 \\
E. coli & 0 & 38.2 & 37.3 & 83.8 \\
\hline
\end{tabular}

${ }^{a}$ SM: standard method; NP: new procedure

the above recommendations. Furthermore, molecular methods are not available in many laboratories, require additional hands-on processing time and costs. Application of MALDI-TOF MS for identification of bacterial colonies from solid media has considerably improved and accelerated routine microbiological diagnostics [11-13]. Particularly, direct MALDI-TOF MS pathogen identification from positive blood culture has been demonstrated to have a positive impact on antimicrobial treatment in septic patients [27, 28]. Most of procedures with this direct approach, however, require additional hands-on processing time. For example, Romero-Gómez et al. used a series of centrifugation, washes and an extraction procedure before identification and AST [3]. Machen et al. used a combined lysisfiltration method [21] whereas Prod'hom et al. used a series of centrifugation, washes and lysis procedure [29]. One major advantage of our combined method is its simplified workflow. Our procedure can be easily integrated in the laboratory routine since it needs only a tube with gel separator (as additional consumable) and a centrifugation step. Identification by MALDI-TOF MS and suspension for AST are performed following a common standard procedure, only earlier than usually (i.e., $3 \mathrm{~h}$ versus overnight incubation).

With respect to rapid identification, our results demonstrate that the performance of the presented method is very high and satisfactory for both Grampositive and Gram-negative isolates. These data confirm the capacity of the MALDI-TOF MS technique to identify both Gram-positive and Gram-negative bacteria from positive blood cultures after a very short-term incubation time, as previously reported [18].

AST also showed excellent results. Percentages of errors (minor, major and very major) were overall low enough to fulfill the performance criteria considered acceptable by the International Standard ISO 20776-2 ( $\mathrm{ME} \leq 3 \%$; $\mathrm{VME} \leq 3 \%$ ). According to previous observations, CoNS exhibited the majority of errors among Gram-positives [3, 21]. These strains, however, are common contaminants of blood cultures that often do not require an antimicrobial treatment. Consequently, the clinical impact of these errors is very low. Among Gram-negative isolates, E. coli had the highest rate of errors but no very major errors occurred. The large number of isolates could have contributed to this result. Similarly, our results indicate a high concordance with regard to detection and/or suggestion of known resistance mechanisms (e.g., production of ESBLs or carbapenemases, methicillin-resistance and ICR). Taken together, these data demonstrate that the rapid method is reliable not only for wild-type isolates (as previously suggested) [3] but also for multidrug-resistant microorganisms.

Finally, in addition to high performance rates for identification and AST, the new procedure exhibited TATs significantly lower than those obtained using the standard method. It is worth noting that the time to positivity was comparable in the two study periods (thus eliminating a confounding variable between the two groups). The processing time was substantially reduced using the new procedure, leading to a final result approximately one day earlier than usual. In this regard, the reduction of TAT was mainly influenced by the automated AST, performed the same day of bottle positivity, even though the application of MALDI-TOF MS technique was crucial to render it possible.

\section{Conclusions}

The new procedure, based on rapid identification by MALDI-TOF MS and automated susceptibility testing performed following short-term incubation cultures on solid agar plates inoculated from positive blood cultures, represents a simple, standardized, and workflow-friendly approach that allows faster and reliable results essentially without additional hands-on processing time and costs. The most relevant conclusion from our experience is that the new procedure permits at least a one day reduction in TAT in the management of bloodstream infections, especially when the most frequently isolated pathogens are involved. The introduction of this method in the microbiology laboratory might facilitate an appropriate species-specific therapy with potential downstream impact on the development of resistance and improved patient outcomes.

\section{Abbreviations}

AST: Antimicrobial susceptibility testing; BSI: Bloodstream infections; CA: Categorical agreement; EA: Essential agreement; MALDI-TOF MS: Matrixassisted laser desorption ionization time-of-flight mass spectrometry; ME: Major error; mE: minor error; TAT: Turnaround time; VME: Very major error

\section{Acknowledgements}

Not applicable

\section{Funding}

No funding

Availability of data and materials

The datasets used and analyzed during the current study are available from the corresponding author on reasonable request. 


\section{Authors' contributions}

CM, study design, laboratory work, collection of data, analysis and interpretation of data, manuscript preparation; SB, EM and NC, study design, laboratory work, collection of data, analysis and interpretation of data; LP and $\mathrm{BP}$, study design, analysis and interpretation of data, critical revision of the manuscript; FL, study design, analysis and interpretation of data, critical revision of the manuscript, final approval of the version to be submitted for publication. All authors read and approved the final manuscript.

\section{Ethics approval and consent to participate}

Not required. Samples were taken as part of the standard patient care and used anonymously.

\section{Consent for publication}

Not required. Our manuscript does not contain data from any individual person.

\section{Competing interests}

The authors declare that they have no competing interests.

\section{Publisher's Note}

Springer Nature remains neutral with regard to jurisdictional claims in published maps and institutional affiliations.

\section{Author details \\ ${ }^{1}$ Microbiology and Virology Unit, A. Manzoni Hospital, Lecco, Italy. ${ }^{2}$ Present address: Clinical Pathology Unit, Vimercate Hospital, Vimercate, Italy.}

\section{Received: 4 April 2017 Accepted: 22 November 2017}

\section{Published online: 06 December 2017}

\section{References}

1. Rhodes A, Evans LE, Alhazzani W, Levy MM, Antonelli M, Ferrer R, et al. Surviving sepsis campaign: international guidelines for Management of Sepsis and Septic Shock: 2016. Intensive Care Med. 2017;43:304-77.

2. Peters RP, van Agtmael MA, Danner SA, Savelkoul PH, Vandenbroucke-Grauls CM New developments in the diagnosis of bloodstream infections. Lancet Infect Dis. 2004:4:751-60.

3. Romero-Gómez MP, Gómez-Gil R, Paño-Pardo JR, Mingorance J. Identification and susceptibility testing of microorganism by direct inoculation from positive blood culture bottles by combining MALDI-TOF and Vitek 2 compact is rapid and effective. J Inf Secur. 2012;65:513-20.

4. Uehara Y, Yagoshi M, Tanimichi Y, Yamada H, Shimoguchi K, Yamamoto S, et al. Impact of reporting gram stain results from blood culture bottles on the selection of antimicrobial agents. Am J Clin Pathol. 2009:132:18-25.

5. Blaschke AJ, Heyrend C, Byington CL, Fisher MA, Barker E, Garrone NF, et al. Rapid identification of pathogens from positive blood cultures by multiplex polymerase chain reaction using the FilmArray system. Diagn Microbiol Infect Dis. 2012;74:349-55.

6. Loonen AJ, Wolffs PF, Bruggeman CA, van den Brule AJ. Developments for improved diagnosis of bacterial bloodstream infections. Eur J Clin Microbiol Infect Dis. 2014;33:1687-702.

7. Christner M, Rohde H, Wolters M, Sobottka I, Wegscheider K, Aepfelbacher M. Rapid identification of bacteria from positive blood culture bottles by use of matrix-assisted laser desorption ionization-time of flight mass spectrometry fingerprinting. J Clin Microbiol. 2010;48:1584-91.

8. Fothergill A, Kasinathan V, Hyman J, Walsh J, Drake T, Wang YF. Rapid identification of bacteria and yeasts from positive-blood-culture bottles by using a lysis-filtration method and matrix-assisted laser desorption ionization-time of flight mass spectrum analysis with the SARAMIS database. J Clin Microbiol. 2013;51:805-9.

9. Eigner U, Holfelder M, Oberdorfer K, Betz-Wild U, Bertsch D, Fahr AM. Performance of a matrix-assisted laser desorption ionization-time-of-flight mass spectrometry system for the identification of bacterial isolates in the clinical routine laboratory. Clin Lab. 2009:55:289-96.

10. Seng P, Drancourt M, Gouriet F, La Scola B, Fournier PE, Rolain JM, et al. Ongoing revolution in bacteriology: routine identification of bacteria by matrix-assisted laser desorption ionization time-of-flight mass spectrometry. Clin Infect Dis. 2009:49:543-51.

11. Bizzini A, Greub G. Matrix-assisted laser desorption ionization time-of-flight mass spectrometry, a revolution in clinical microbial identification. Clin Microbiol Infect. 2010;16:1614-9.
12. Cherkaoui A, Hibbs J, Emonet S, Tangomo M, Girard M, Francois P, et al. Comparison of two matrix-assisted laser desorption ionization-time of flight mass spectrometry methods with conventional phenotypic identification for routine identification of bacteria to the species level. J Clin Microbiol. 2010; 48:1169-75.

13. van Veen SQ, Claas EC, Kuijper EJ. High-throughput identification of bacteria and yeast by matrix-assisted laser desorption ionization time-of-flight mass spectrometry in conventional medical microbiology laboratories. J Clin Microbiol. 2010:48:900-7.

14. Ferreira L, Sánchez-Juanes F, González-Avila M, Cembrero-Fuciños D, Herrero-Hernández A, González-Buitrago JM, et al. Direct identification of urinary tract pathogens from urine samples by matrix-assisted laser desorption ionization-time of flight mass spectrometry. J Clin Microbiol. 2010;48:2110-5

15. Schubert S, Weinert K, Wagner C, Gunzl B, Wieser A, Maier T, et al. Novel, improved sample preparation for rapid, direct identification from positive blood cultures using matrix-assisted laser desorption/ionization time-offlight (MALDI-TOF) mass spectrometry. J Mol Diagn. 2011;13:701-6.

16. Drancourt M. Detection of microorganisms in blood specimens using matrix-assisted laser desorption ionization time-of-flight mass spectrometry: a review. Clin Microbiol Infect. 2010;16:1620-5.

17. Farina C, Arena F, Casprini P, Cichero P, Clementi M, Cosentino M, et al. Direct identification of microorganisms from positive blood cultures using the lysis-filtration technique and matrix assisted laser desorption ionization time-of-flight mass spectrometry (MALDI-TOF MS): a multicentre study. New Microbiol. 2015:38:245-50.

18. Idelevich EA, Schüle I, Grünastel B, Wüllenweber J, Peters G, Becker K. Rapid identification of microorganisms from positive blood cultures by MALDITOF mass spectrometry subsequent to very short-term incubation on solid medium. Clin Microbiol Infect. 2014:20:1001-6.

19. La Scola B, Raoult D. Direct identification of bacteria in positive blood culture bottles by matrix-assisted laser desorption ionisation time-of-flight mass spectrometry. PLoS One. 2009;25:e8041.

20. Stevenson LG, Drake SK, Murray PR. Rapid identification of bacteria in positive blood culture broths by matrix-assisted laser desorption ionizationtime of flight mass spectrometry. J Clin Microbiol. 2010;48:444-7.

21. Machen A, Drake T, Wang YF. Same day identification and full pane antimicrobial susceptibility testing of bacteria from positive blood culture bottles made possible by a combined lysis-filtration method with MALDI-TOF VITEK mass spectrometry and the VITEK 2 system. PLoS One. 2014:14:e87870.

22. Wattal C, Oberoi JK. Microbial identification and automated antibiotic susceptibility testing directly from positive blood cultures using MALDI-TOF MS and VITEK 2. Eur J Clin Microbiol Infect Dis. 2016:35:75-82.

23. EUCAST 2014. EUCAST clinical breakpoints v.4. European committee on antimicrobial susceptibility testing. http://www.eucast.org/fileadmin/src/ media/PDFs/EUCAST_files/Breakpoint_tables/Breakpoint_table_v_4.0.pdf

24. EUCAST 2015. EUCAST clinical breakpoints v.5. European committee on antimicrobial susceptibility testing. http://www.eucast.org/ fileadmin/src/media/PDFs/EUCAST files/Breakpoint_tables/v_5.0 Breakpoint_Table_01.pdf

25. ISO 20776-2 (2007). Clinical laboratory testing and in vitro diagnostic test systems - Susceptibility testing of infectious agents and evaluation of performance of antimicrobial susceptibility test devices - Part 2: Evaluation of performance of antimicrobial susceptibility test devices. http://www.iso.org/iso/catalogue_detail.htm?csnumber=41631.

26. Kumar A, Roberts D, Wood KE, Light B, Parrillo JE, Sharma S, et al. Duration of hypotension before initiation of effective antimicrobial therapy is the critical determinant of survival in human septic shock. Crit Care Med. 2006; 34:1589-96

27. Clerc O, Prod'hom G, Vogne C, Bizzini A, Calandra T, Greub G. Impact of matrix-assisted laser desorption ionization time-of-flight mass spectrometry on the clinical management of patients with gram-negative bacteremia: a prospective observational study. Clin Infect Dis. 2013;56:1101-7.

28. Vlek AL, Bonten MJ, Boel CH. Direct matrix-assisted laser desorption ionization time-of-flight mass spectrometry improves appropriateness of antibiotic treatment of bacteremia. PLoS One. 2012;7:e32589.

29. Prod'hom G, Durussel C, Greub G. A simple blood-culture bacterial pellet preparation for faster accurate direct bacterial identification and antibiotic susceptibility testing with the VITEK 2 system. J Med Microbiol. 2013;62:773-7. 